\title{
An Artificial Iris ASIC with High Voltage Liquid Crystal Driver and 10nA Light Range Detector and 40nA Blink Detector for LCD flicker removal
}

\author{
Bogdan C. Raducanu ${ }^{1}$, Samira Zali Asl², Stefano Stanzione ${ }^{2}$, Chris van Liempd ${ }^{2}$, Andrés Vásquez Quintero $^{3}$, \\ Herbert De Smet ${ }^{3}$, Johan De Baets ${ }^{3}$, Chris van Hoof ${ }^{1,4}$, Nick Van Helleputte ${ }^{1}$ \\ ${ }^{1}$ imec, Leuven, Belgium, ${ }^{2}$ imec/Holst Centre, Eindhoven, the Netherlands, ${ }^{3} \mathrm{CMST} /$ Ghent University, Ghent, Belgium, \\ ${ }^{4}$ KU Leuven, Heverlee, Belgium \\ raducanu@imec.be
}

\begin{abstract}
In a functional eye, the iris controls the pupil diameter to regulate the exposure of the retina. While iris deficiencies such as aniridia or leiomyoma can be mitigated with fixed or adaptive artificial irises [1] and adaptive transparency glasses exist to alleviate this situation, they do not mimic the normal functionality of the natural iris. To address this, a fully encapsulated, self-contained iris embedded in a smart contact lens is proposed. Keywords: contact lens, aniridia, artificial, iris, light readout, blink detector, LCD
\end{abstract}

\section{Proposed solution}

Fig. 1 shows the artificial iris contact lens and block diagram comprising an embedded guest-host LCD, a control ASIC and embedded near field communication (NFC) powering. The electronics are interconnected using thin film and embedded in a thermoplastic carrier which will be thermoformed to achieve the required curvature to fit the eye shape [2]. To ensure the uniform separation, these guest-host liquid crystal is held together by a matrix of spacer structures [3] between two flexible substrates The variable iris aperture is implemented with 4 concentric rings on the LCD [3] which turn opaque depending on ambient light levels measured by the ASIC using integrated photodiodes (PD). A $\mu \mathrm{Wh}$ level consumption is required to run the lens for a full day, which is powered by an embedded 3-3.6V power source and charged overnight. The primary function of the ASIC is to generate the appropriate LCD control signals based on ambient light levels using a few core blocks: an ambient light range detector (LRD), an eye blink detector (BD) and an LCD driver.

An ultra-low-power ambient light range detector quantizes the incoming light into 4 consecutive light ranges to control the 4-ring iris. The naked die embedded into the lens uses an integrated PD to measure the light. Typically, a transimpedance-based readout is too power-hungry in high light-level conditions (>100klux) and alternatively measuring the PD open voltage has a high PVT spread and very low signal levels under low-light conditions (1lux). Therefore, to achieve a low-power solution covering the $>5$ decades of light intensity, the LRD in Fig. 2 is used. The photodiode current $\mathrm{IPD}_{\mathrm{PD}}$ is successively compared to an increasing and programmable IDAC current, starting at the lowest level (400pA for $\sim 6.4$ lux) and continuing through the 4 levels until the comparator indicates $\mathrm{I}_{\mathrm{PD}}>\mathrm{I}_{\mathrm{DAC}}\left(\mathrm{V}_{\mathrm{OUT}}<0\right)$. A simple comparator with $0 \mathrm{~V}$ reference can be used, since the input will saturate to either -VOP or to VDD. Adequate timing accounts for the large PD capacitance $\left(\mathrm{C}_{\mathrm{PD}} \sim 19 \mathrm{pF}\right)$ and a $4.8 \mathrm{kHz}$ and an $80 \mathrm{kHz}$ clocks are used alternatively to minimize the power in different light ranges. This wide range LRD consumes an average of $\sim 10 \mathrm{nA}$ under office light levels with $43 \mathrm{mSec}$ conversion time. To account for user and ASIC variability, a 4-bit Trim setting is available for each of the I IAC currents. The impact of artificial light flicker $(50 / 60 \mathrm{~Hz})$ and eye blinks (nominal duration $<200 \mathrm{mSec}$ ) is removed by using the digital maximum detector monitoring the maximum readout output out of 12 samples $(12 \times 43 \mathrm{mSec}>0.5 \mathrm{Sec})$.

Based on the LRD output, the LCD driver turns on the correct amount of LCD rings. The LCD elements require $>10 \mathrm{Vpp}$ bias voltage. 4 charge pumps $\left(\mathrm{V}_{\mathrm{CP}}\right)$ are used, with the LCD itself acting as the storage capacitor (Fig. 3). As shown in [4], the LCDs needs AC drive signal to have reasonable lifespan. To save power, low frequency AC avoids high power consumption of high frequency $\mathrm{AC}$ and drastically short live of DC driving. Direct-polarity driving is achieved with a grounded common electrode and a $\mathrm{V}_{\mathrm{CP}}$ applied to the required segments. In reverse polarity driving, $\mathrm{V}_{\mathrm{CP}}$ is applied to the common electrode using 4 parallel charge pumps while the opaque segments are grounded.

To make the flicker due to LCD transition time unperceivable, the LCD polarity switching is aligned with eye blinks. A 14 second fail-safe timeout is used in case blinks are missed or not happened. Since the eye lid blocks $\sim 95 \%$ of the incoming light, an eye blink detector circuit (Fig. 4) detects a sudden light level drop as opposed to a single absolute $\mathrm{I}_{\mathrm{PD}}$ threshold. Hence $\mathrm{I}_{\mathrm{PD}}$ is fed into a logarithmic current-to-voltage converter created by the diode-feedback amplifier which generates an output voltage proportional to the relative variation. Only fast signals filtered by the high-pass filter reach the comparator and are detected. For enough speed despite the high $\mathrm{C}_{\mathrm{PD}}$, the $\mathrm{PD}$ is biased with a constant voltage bias. Under low light, a larger PD is used to provide enough sensitivity, while in brighter scenes a smaller PD is used to save power. Under typical indoor lighting, the eye blink detection circuit consumes 40nA.

The ASIC was fabricated in a $0.18 \mu \mathrm{m} 16 \mathrm{~V}$ BCD TSMC technology. Fig. 5 shows the ASIC micrograph and key performance measurements. The response of the emulated pupil diameter is compared to a natural one at typical thresholds. Given personal preference, each of the thresholds is tunable (1.2lux to 4.1klux). The total current consumption under typical indoor light levels ( 2 segments on) is 730nA and drops to $62 \mathrm{nA}$ in near dark (all segments off). Fig. 5 also shows a comparison table with ASICs containing similar functions: an ambient light detector [5] and a contact lens LCD driver [3]. The BD was tested with a controllable light source and it reliably triggers when the illuminance drops at least $65 \%$ 
during 50ms (measured over 15-5klux), corresponding to the expected shielding provided by the human eye lid and speed of human blink. The LCD driver achieves typ. 13Vpp drive with $3 \mathrm{~ms}$ rise time for $3 \mathrm{~V}$ supply.

This work demonstrates a fully integrated artificial iris in a contact lens form factor, providing a variable aperture using a 4-ring LCD. The demonstrated ASIC contains a $\mathrm{nW}$ power light range detector with on-chip photodiodes to automatically limit the amount of light entering the eye by appropriately darkening the respective LCD rings using charge pumps. A low frequency LCD driving method is used where the polarity switching is aligned with the blinks, ensuring a 730nA typ. current consumption.

\section{References}

[1] J. De Smet, A. Avci, R. Beernaert, D. Cuypers, and H. De Smet, "Design and wrinkling behavior of a contact lens with an integrated liquid crystal light modulator," IEEE/OSA J. Disp. Technol., vol. 8, no. 5, pp. 299-305, 2012.

[2] A. Vásquez Quintero, R. Verplancke, H. De Smet, and J. Vanfleteren, "Stretchable Electronic Platform for Soft and Smart Contact Lens Applications," Adv. Mater. Technol., vol. 2, no. 8, 2017.

[3] F. De Roose et al., "An active artificial iris controlled by a $25-\mu \mathrm{W}$ flexible thin-film driver," in Technical Digest - International Electron Devices Meeting, IEDM, 2017, pp. 32.1.1-32.1.4.

[4] J. Pirš, D. Ponikvar, B. Marin, and M. Chambers, "Low power consumption driving for liquid crystal light modulators," Displays, vol. 25, no. 4, 2004.

[5] S. Sengupta, H. Ouh, and M. L. Johnston, "An all-digital CMOS ambient light sensor using a single photon avalanche diode," in Proceedings of IEEE Sensors, 2017, vol. 2017-Decem.

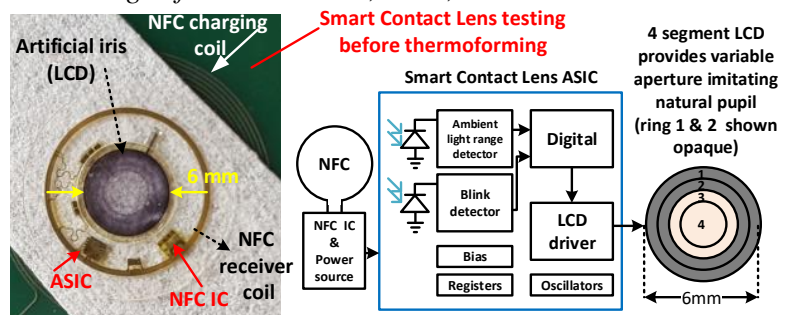

Fig. 1 Left: smart contact lens solution in flat format and right: top level block diagram of the full solution with a 4 ring LCD.

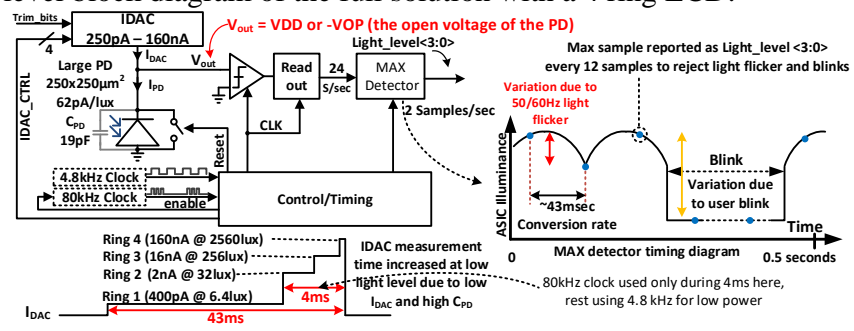

Fig. 2 Light range detector block diagram. IPD is successively compared with 4 level IDAC. MAX detector rejects the light flickering and blink events.

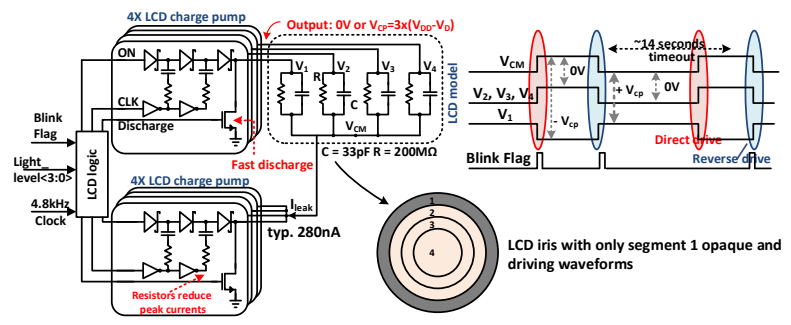

Fig. 3 LCD driver consisting of 8 charge pumps using high voltage components to boost the voltage and iris LCD showing the first segment opaque along with associated drive waveforms, indicating the polarity reversal due to eye blinks or timeout.
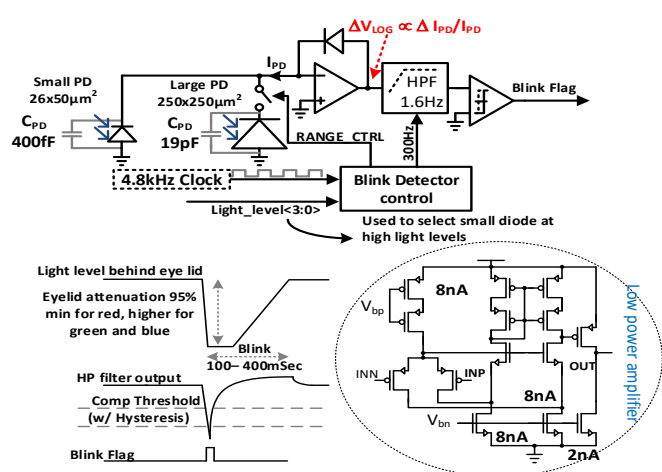

Fig. 4 Blink detector block and timing diagram. The log amplifier in the feedback outputs a voltage proportional to the relative variation of light level, it is high pass filtered to detect a blink event.

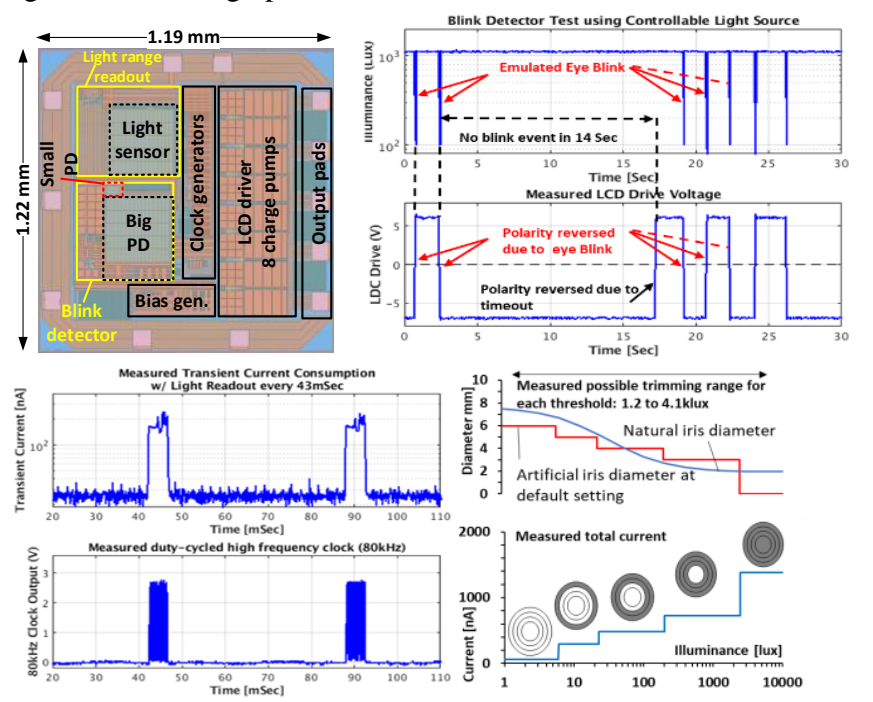

\begin{tabular}{|c|c|c|c|}
\hline & {$[2]$} & {$[4]$} & $\begin{array}{c}\text { This } \\
\text { work }\end{array}$ \\
\hline LCD segments & 1 & - & $\mathbf{4}$ \\
\hline Supply range [V] & $5-9$ & 1 & $\mathbf{3 - 3 . 6}$ \\
\hline Power consumption (office light) [W] & - & $125 \mu$ & $\mathbf{1 8 4 n}$ \\
\hline Active power 1/2/3/4 segments $[\mu \mathrm{W}]$ & 25 & - & $\begin{array}{c}\mathbf{0 . 9 3 / 1 . 9 /} \\
\mathbf{2 . 2} / \mathbf{4 . 2}\end{array}$ \\
\hline LCD drive [Vpp] & 10 & - & $\mathbf{1 3}$ \\
\hline LCD flicker removal & No & - & Yes \\
\hline Ambient light range [lux] & - & $100-25 \mathrm{k}$ & $\mathbf{1 . 2 - 1 9 0 k}$ \\
\hline Blink sensitivity, max [\%] & - & - & $\mathbf{- 6 5}$ \\
\hline Active chip area $\left[\mathrm{mm}^{2}\right]$ & 0.75 & 0.046 & $\mathbf{1 . 4 5}$ \\
\hline Technology node $[\mu \mathrm{m}]$ & $5 \mu \mathrm{m}$ & 0.13 & $\mathbf{0 . 1 8 \mu m}$ \\
& TFT & CMOS & BCD \\
\hline
\end{tabular}

Fig. 5 Die micrograph and key performance measurements: blink detector test with controllable light source and LCD output voltage showing polarity reversal at blinks and timeout; measured power consumption of chip during ambient light level readout showing $80 \mathrm{kHz}$ clock duty cycled usage; measured artificial pupil diameter and table with measured performance compared with artificial lens LCD driver and ambient light sensor. 